Research Paper

\title{
Dynamic Compression Effects on Immature Nucleus Pulposus: a Study Using a Novel Intelligent and Me- chanically Active Bioreactor
} \author{
Sukai $\mathrm{Li}^{1}$, Yangbin $\mathrm{Ou}^{1}$, Qiang Zhou ${ }^{1}$ \\ 1. Department of Orthopedic Surgery, Southwest Hospital, Third Military Medical University, Chongqing, 400038, China; \\ 2. Department of Orthopedic Surgery, Chongqing Three Gorges Central Hospital, Chongqing, 404000, China; \\ 3. Department of Orthopedic Surgery, Xinqiao Hospital, Third Military Medical University, Chongqing, 400038, China; \\ 4. Department of Orthopedic Surgery, No. 181 Hospital of PLA, Guilin, Guangxi, 541002, China. \\ $\bowtie$ Corresponding author: E-mail: zq_tlh@163.com (Qiang Zhou).
}

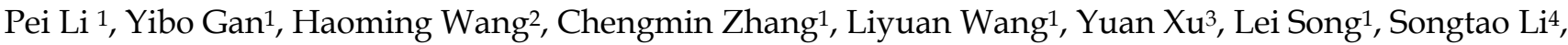

(1) Ivyspring International Publisher. Reproduction is permitted for personal, noncommercial use, provided that the article is in whole, unmodified, and properly cited. See http://ivyspring.com/terms for terms and conditions.

Received: 2015.09.04; Accepted: 2016.01.22; Published: 2016.02.20

\begin{abstract}
Background: Previous cell culture and animal in vivo studies indicate the obvious effects of mechanical compression on disc cell biology. However, the effects of dynamic compression magnitude, frequency and duration on the immature nucleus pulposus (NP) from an organ-cultured disc are not well understood.

Objective: To investigate the effects of a relatively wide range of compressive magnitudes, frequencies and durations on cell apoptosis and matrix composition within the immature NP using an intelligent and mechanically active bioreactor.

Methods: Discs from the immature porcine were cultured in a mechanically active bioreactor for 7 days. The discs in various compressive magnitude groups $(0.1,0.2,0.4,0.8$ and $1.3 \mathrm{MPa}$ at a frequency of 1.0 $\mathrm{Hz}$ for 2 hours), frequency groups $(0.1,0.5,1.0,3.0$ and $5.0 \mathrm{~Hz}$ at a magnitude of $0.4 \mathrm{MPa}$ for 2 hours) and duration groups $(1,2,4$ and 8 hours at a magnitude of $0.4 \mathrm{MPa}$ and frequency of $1.0 \mathrm{~Hz})$ experienced dynamic compression once per day. Discs cultured without compression were used as controls. Immature NP samples were analyzed using the TUNEL assay, histological staining, glycosaminoglycan (GAG) content measurement, real-time PCR and collagen II immunohistochemical staining.

Results: In the $1.3 \mathrm{MPa}, 5.0 \mathrm{~Hz}$ and 8 hour groups, the immature NP showed a significantly increase in apoptotic cells, a catabolic gene expression profile with down-regulated matrix molecules and up-regulated matrix degradation enzymes, and decreased GAG content and collagen II deposition. In the other compressive magnitude, frequency and duration groups, the immature NP showed a healthier status regarding NP cell apoptosis, gene expression profile and matrix production.

Conclusion: Cell apoptosis and matrix composition within the immature NP were compressive magnitude-, frequency- and duration-dependent. The relatively high compressive magnitude or frequency and long compressive duration are not helpful for maintaining the healthy status of an immature NP.
\end{abstract}

Key words: intervertebral disc degeneration, immature, nucleus pulposus, organ culture, bioreactor, dynamic compression.

\section{Introduction}

Low back pain (LBP) is a chronic condition worldwide with a high lifetime prevalence [1]. Mounting epidemiological evidence and basic research indicate a close relationship between LBP and intervertebral disc degeneration (IDD) [2]. To date, the accurate biological pathways contributing to disc degeneration remain unclear.

Previous studies demonstrated that mechanical load is necessary for intervertebral disc (IVD) development and disc matrix homeostasis, whereas inap- 
propriate mechanical load plays an important role in initiating and/or aggravating disc degeneration [3]. During the last decade, several studies investigated the responses of the disc cell to mechanical stimulation in artificial three-dimensional culture [4, 5]. However, removal of the native extracellular matrix eliminates certain mechanotransduction pathways, which may have practical implications under physiological conditions [6]. By contrast, in vivo animal studies can maintain the physiological environments of the surrounding disc cells. These in vivo studies including rat tail and mouse tail models revealed extensive information on disc mechanobiology by applying an external load [7, 8]. However, the loading pattern in these rodent coccygeal discs may be quite different from that in human discs [6].

The disc/endplate organ culture is regarded as a good model to study nucleus pulposus (NP) biology due to its precise controllability over external stimuli and its retention of native structural integrity [9]. In particular, the development of a bioreactor platform can further maintain NP viability for a long period, and some studies can be performed at a near physiological condition. Previously, several studies [6, 10, 11] assessed the effects of several mechanical parameters on NP cells using the disc bioreactor culture model and provided a wealth of information about the interplay between certain mechanical parameters and NP metabolism. In our preliminary study, we developed an intelligent and mechanically active perfusion bioreactor combined with a substance exchanger [12]. Compared to other bioreactors used for disc organ culture $[6,10,13]$, the main advantage of this perfusion bioreactor is that it can automatically control the culture environment including the $\mathrm{pH}$, $\mathrm{PO}_{2}$, glucose and lactic acid. These parameters can affect on NP biology in vitro [14]. Therefore, a more advanced and stable bioreactor system may further improve our understanding of NP mechanobiology in vitro.

In humans, the original notochordal cells within the NP tissue disappears around the age of 10 [15]. Moreover, previous studies indicated that notochordal cells can protect the disc from degeneration, which supports the finding that the first signs of disc degeneration simultaneously occurr with the disappearance of the notochordal cells [16, 17]. Therefore, the immature human disc may be the most appropriate model to study the initiating stage of disc degeneration. However, it is unrealistic to obtain abundant immature human discs because of some ethical limitations. Porcine is accepted as another suitable large animal model for investigating disc structure, biochemistry and biomechanics [13]. Furthermore, immature porcine discs have a high content of noto- chordal cells [18], which is similar to that of immature human discs. Therefore, we propose that investigations on immature porcine discs may have merits by reflecting biological changes of the initial stage of disc degeneration.

The effects of mechanical load on NP biology are magnitude-, frequency- and duration-dependent due to the viscoelasticity and creep properties of discs [19]. In the present study, we used the intelligent and mechanically active bioreactor culture system to study the effects of a relatively wide range of dynamic compressive magnitudes (0.1-1.3 $\mathrm{MPa})$, frequencies (0.1-5.0 Hz) and durations (1-8 hours per day) on cell apoptosis and matrix composition within the immature NP. The immature NP samples were analyzed for histology, cell apoptosis, gene expression and matrix composition.

\section{Materials and methods}

\section{Intervertebral disc harvest}

As described [20], discs (T11-L5) with cartilage endplate (CEP) were harvested from fourteen healthy immature pigs (3-4 months old) under sterile conditions. Subsequently, the disc area was measured to calculate the compressive magnitude based on the equation: Area $\approx \Pi\left(W_{\text {ap }} W_{\text {lat }}\right) / 4$, where $W_{\text {ap }}$ and $W_{\text {lat }}$ are the anterior-posterior and lateral widths, respectively [21]. All animal experiments were approved by the Ethics Committee at Southwest Hospital affiliated to the Third Military Medical University [SYXK (YU) 2012-0012].

\section{Bioreactor design}

As illustrated in Figure 1, the perfusion bioreactor primarily consists of a medium reservoir, peristaltic pump, substance exchanger, $\mathrm{pH}$ sensor, $\mathrm{PO}_{2}$ sensor, $\mathrm{PCO}_{2}$ sensor, tissue culture chamber, loading application device and other ancillary equipment. Mechanical loading is axially applied with an integrated servomotor mated with the culture chamber and simultaneously adjusted by a central controller. The medium perfusion system includes two circulating loops, an incubation loop and a medium supplement loop. The fresh medium in the medium supplement loop can be recycled into the medium reservoir after following into the substance exchanger. Additional details about this bioreactor system were reported previously [12].

\section{Disc organ culture and loading frame}

Discs were randomly assigned to different compressive magnitude groups $(0.1,0.2,0.4,0.8$ and 1.3 $\mathrm{MPa}$ at a frequency of $1.0 \mathrm{~Hz}$ for 2 hours per day), compressive frequency groups $(0.1,0.5,1.0,3.0$ and $5.0 \mathrm{~Hz}$ at a magnitude of $0.4 \mathrm{MPa}$ for 2 hours per day) 
and compressive duration groups $(1,2,4$ and 8 hours per day at a magnitude of $0.4 \mathrm{MPa}$ and frequency of $1.0 \mathrm{~Hz}$ ). The unloaded discs were used as controls. DMEM media (high glucose, Hyclone) containing 1\% (v/v) penicillin-streptomycin, $10 \%(\mathrm{v} / \mathrm{v})$ fetal bovine serum (FBS, Gibco) and $0.025 \mathrm{mg} / \mathrm{mL}$ ascorbic acid (Sigma) was circulated at $15 \mathrm{~mL} / \mathrm{min}$ for 7 days and changed when needed. The medium osmolarity was increased to $430 \mathrm{mOsm} / \mathrm{kg}$ using sodium chloride and verified with a freezing-point osmometer. The $\mathrm{pH}$ value was adjusted to 7.2 with $\mathrm{HCl}$ and $\mathrm{NaCl}$. When the substance exchanger was turned on, a $\mathrm{pH}$ of 7.2-7.4 and a $\mathrm{PO}_{2}$ of $160-180 \mathrm{mmHg}$ in the $\mathrm{CO}_{2}$ incubator were manually set at the digital controller. At the end of the culture period, the NP samples were isolated under a dissecting microscope and used for subsequent analyses.

\section{Histological analysis}

Discs were fixed with $4 \%$ paraformaldehyde, decalcified with $10 \%$ ethylenediaminetetraacetic acid (EDTA) and embedded in paraffin. Then, $5 \mu \mathrm{m}$ thick cross-sections were prepared. To observe NP cell morphology and proteoglycan (PG) distribution within the immature NP tissue, hematoxylin and eosin (HE) staining and alcian blue staning were respectively performed. All sections were observed under a light microscopy (Olympus BX51).

\section{Measurement of NP cell apoptosis}

NP cell apoptosis was investigated by terminal deoxynucleotidyl transferase dUTP nick-end labeling (TUNEL) assay. Briefly, disc sections were dewaxed and permeated with proteinase $K$, and then TUNEL staining was performed with an In Situ Cell Death Detection Kit (Roche) according to the instructions. Negative control in which label solution replaced TUNEL reaction mix was also used. NP cell apoptosis was calculated as the percentage of TUNEL-positive NP cells to total NP cells.

\section{Real-time PCR analysis}

Gene expression of matrix molecules (aggrecan and collagen II) and matrix remodeling enzymes (TIMP-1, TIMP-3, ADAMTS-4 and MMP-3) was analyzed by real-time PCR. Briefly, after total RNA was extracted from NP sample with TRIzol solution (Invitrogen) and synthesized into complementary DNA (cDNA) with a reverse transcription kit (Roche), the reaction system containing specific primers, cDNA and SYBR Green qPCR Mix (DONGSHENG BIOTECH, China) was subjected to a real-time PCR system. Primers of genes (Table 1) were synthesized by a biological company (Sangon, Biotech Co., Ltd., China). GAPDH was used as the reference gene and expression of target genes was calculated as $2^{-\Delta \Delta C t}$.

Table 1. Primers of target genes.

\begin{tabular}{llll}
\hline Gene & Accession number & Forward $\left(5^{\prime}-3^{\prime}\right)$ & Reverse $\left(5^{\prime}-3^{\prime}\right)$ \\
\hline GAPDH & NM_001206359.1 & ACCTCCACTACATGGTCTACA & ATGACAAGCTTCCCGTTCTC \\
Aggrecan & NM_001164652.1 & CGTGGTCCAGCACTTCTAAA & AGTCCACTGAGATCCTCTACTC \\
Collagen II & XM_001925959.4 & CCGGGTGAACGTGGAGAGACTG & CGCCCCCACAGTGCCCTC \\
ADAMTs-4 & XM_003481414.2 & TTCAACGCCACGTTCTACTC & GCCGGGATGATGAGGTTATTT \\
MMP-3 & NM_001166308.1 & GCCCGTTGAGCCCACAGAATCTAC & GGAAGAGGTGGCCAAAATGAAGAG TI \\
MP-1 & NM_213857.1 & CCTGACATCCGGTTCATCTA & CAGTTGTCCAGCTATGAGAAAC \\
TIMP-3 & XM_003126073.4 & GGATTGTGTAACTTTGTGGAGAG & GGCAGGTAGTAGCAGGATTTA \\
\hline
\end{tabular}
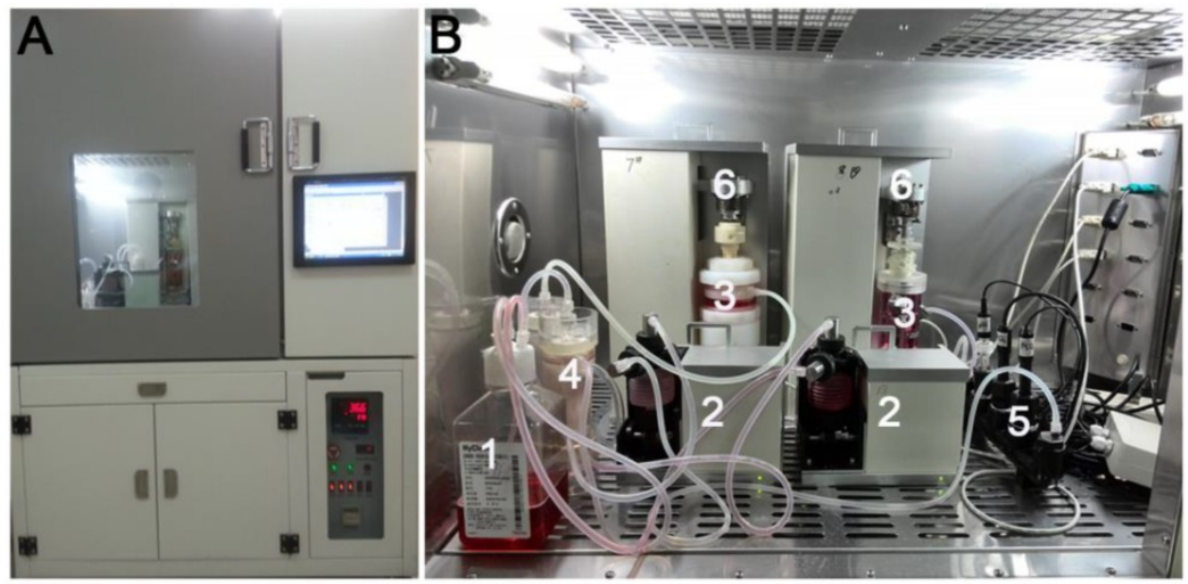

Figure 1. Schematic of bioreactor system for culturing discs. (A) Overview image of the bioreactor platform. (B) Primary units of the bioreactor system (1: medium reservoir; 2: peristaltic pump; 3: tissue culture chamber; 4: substance exchanger; 5: $\mathrm{pH}, \mathrm{PO}_{2}$ and $\mathrm{PCO}_{2}$ sensor; 6: loading application device). 


\section{Quantification of glycosaminoglycans (GAG) content}

Briefly, after NP samples were lyophilized for 24 hours, the dried NP samples were digested with papain solution. Then, GAG content normalized to the tissue dry weight was determined using dimethylmethylene blue (DMMB) assay [22].

\section{Antibodies and immunohistochemistry}

To analyze collagen II protein expression within the immature $\mathrm{NP}$, immunohistochemical staining was performed on disc sections as described [23]. The primary antibody used in this study was mouse anti-collagen II (Abcam, diluted 1:200). After color development with diaminobenzidine, all disc sections were observed under a light microscopy (Olympus BX51).

\section{Statistics}

The numerical data were expressed as mean \pm SD and statistical analysis was performed using SPSS 13.0 software. When homogeneity test for variance was completed, comparison between two groups was analyzed by Independent-Samples T test. A statistical difference was indicated when $\mathrm{p}$-value $<0.05$.

\section{Results}

\section{Histology}

After loading different compressive magnitudes, frequencies or durations, no obvious changes within the immature NP were found by HE staining compared with the control group (Figure 2A-C). Alcian blue staining indicated that the PG content in all compressive groups except the $1.3 \mathrm{MPa}, 5.0 \mathrm{~Hz}$ and 8 hours groups remained nearly constant or increased compared with the control group (Figure 2A-C).

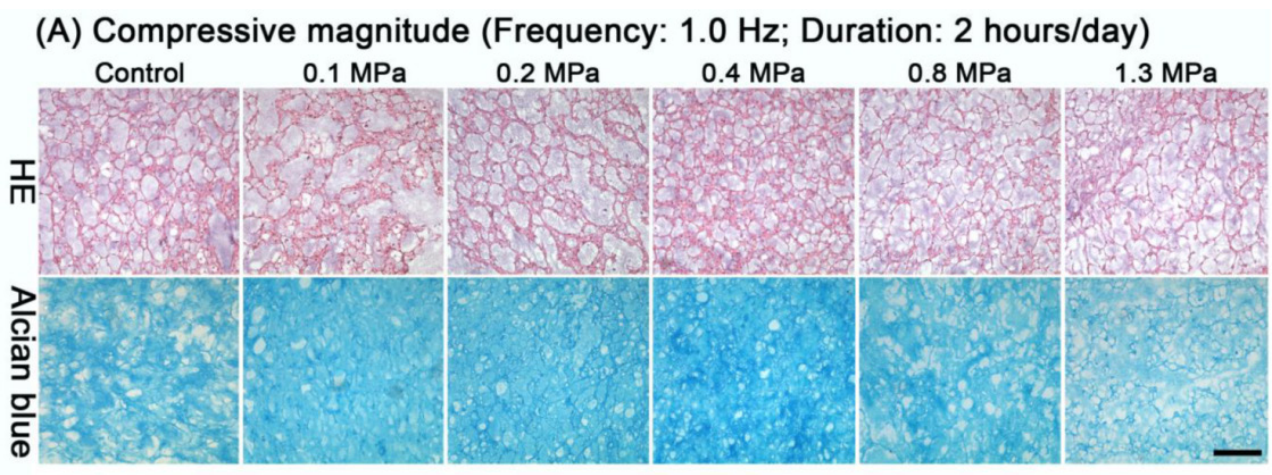

(B) Compressive frequency (Magnitude: $0.4 \mathrm{MPa}$; Duration:2 hours/day)

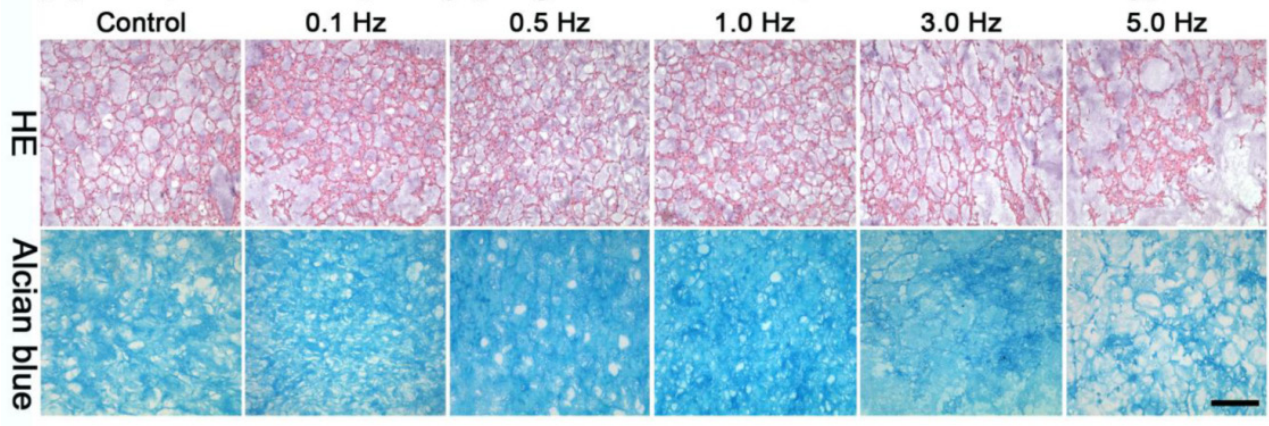

(C) Compressive duration (Magnitude: $0.4 \mathrm{MPa}$; Frequency: $1.0 \mathrm{~Hz}$ )

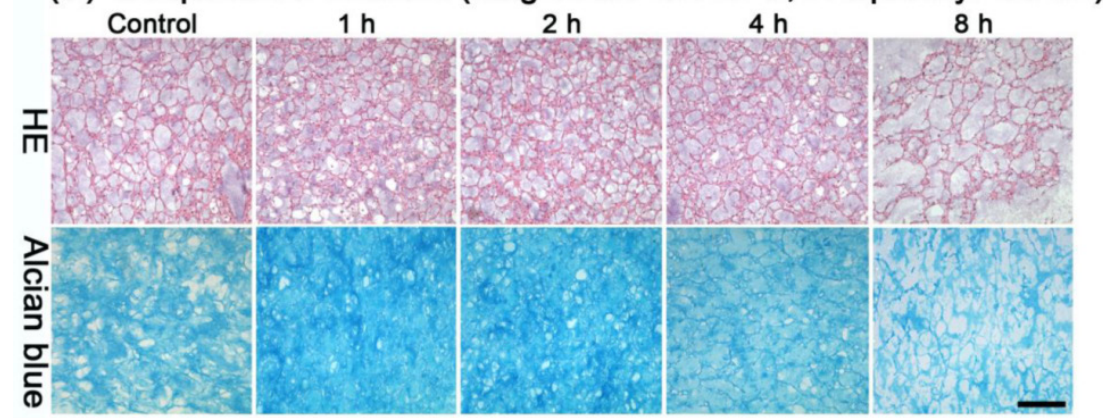

Figure 2. Histological analysis of immature nucleus pulposus (NP). HE staining and alcian blue staining of immature NP from discs in the different compressive magnitude groups (A), compressive frequency groups (B) and compressive duration groups (C). Magnification: $200 x ; s c a l e=100 \mu m ; n=3$. 
(A) Compressive magnitude (Frequency: $1.0 \mathrm{~Hz}$; Duration: 2 hours/day)

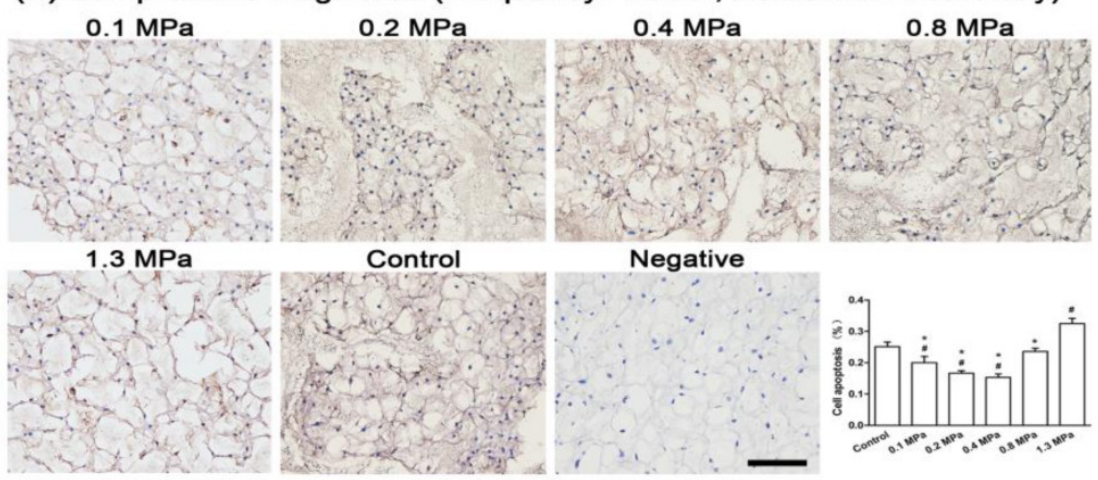

(B) Compressive frequency (Magnitude: $0.4 \mathrm{MPa}$; Duration:2 hours/day)

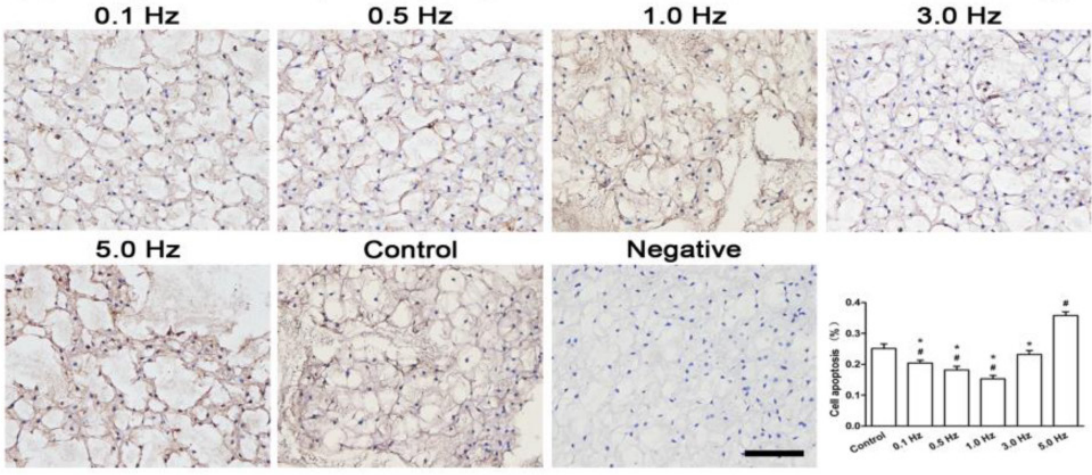

(C) Compressive duration (Magnitude: $0.4 \mathrm{MPa}$; Frequency: $1.0 \mathrm{~Hz}$ )

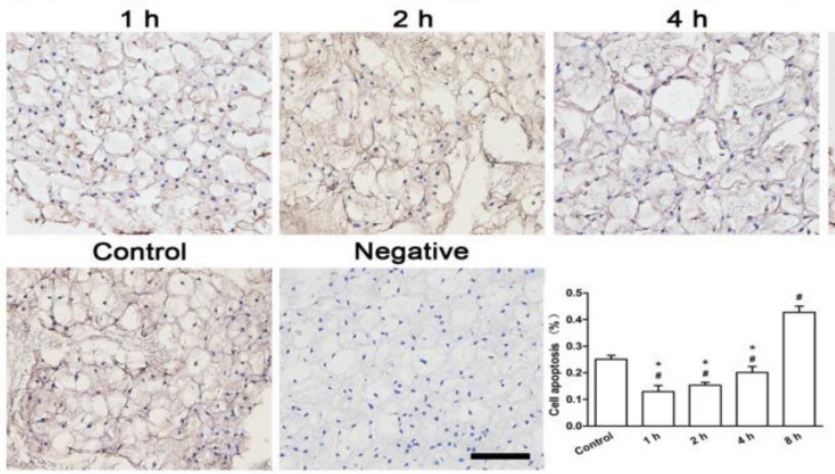

Figure 3. Cell apoptosis in immature nucleus pulposus (NP). Terminal deoxynucleotidyl transferase dUTP nick-end labeling (TUNEL) staining of immature NP and its quantitative analysis in different compressive magnitude groups (A), compressive frequency groups (B) and compressive duration groups (C). Magnification: 200x; scale $=100 \mu \mathrm{m} ; n=3$. Data are expressed as the means $\pm S D, n=3$. \#: Indicates a significant difference $(p<0.05)$ compared with the control group $(p<0.05)$ between two groups. *: Indicates a significant difference $(\mathrm{p}<0.05)$ compared with the compression groups $(1.3 \mathrm{MPa}, 5 \mathrm{~Hz}$ or 8 hour).

\section{NP cell apoptosis}

Apoptotic NP cells appeared in all compressive groups. For the compressive magnitude groups (Figure 3A), apoptotic NP cells were decreased in the 0.1 $\mathrm{MPa}, 0.2 \mathrm{MPa}$ and $0.4 \mathrm{MPa}$ groups, increased in the $1.3 \mathrm{MPa}$ group and nearly unchanged in the $0.8 \mathrm{MPa}$ group compared with the control group. For the compressive frequency groups (Figure 3B), apoptotic $\mathrm{NP}$ cells were decreased in the $0.1 \mathrm{~Hz}, 0.5 \mathrm{~Hz}$ and 1.0 $\mathrm{Hz}$ groups, unchanged in the $3.0 \mathrm{~Hz}$ group and significantly increased in the $5.0 \mathrm{~Hz}$ group compared with the control group. For the compressive duration groups (Figure 3C), apoptotic NP cells were increased with increasing duration to a maximum in the 8 hour group.

\section{Gene expression}

Gene expression was significantly influenced by different compressive magnitudes, frequencies and durations. For the compressive magnitude groups (Figure 4A), the expression of matrix genes (aggrecan and collagen II) was up-regulated in the $0.1 \mathrm{MPa}, 0.2$ $\mathrm{MPa}, \quad 0.4 \mathrm{MPa}$ and $0.8 \mathrm{MPa}$ groups and down-regulated in the $1.3 \mathrm{MPa}$ group compared with the control group, whereas the expression of catabolic genes (ADAMTS-4 and MMP-3) showed an opposite trend with the majority up-regulated in the $1.3 \mathrm{MPa}$ 
group. TIMP-1 expression was not significantly changed among the compressive magnitude groups and control group. A larger fold change of TIMP-3 expression was observed in the $0.1 \mathrm{MPa}, 0.2 \mathrm{MPa}$ and $0.4 \mathrm{MPa}$ groups compared with the control group.

For the compressive frequency groups (Figure 4B), the expression of matrix genes (aggrecan and collagen II) in the $0.1 \mathrm{~Hz}, 0.5 \mathrm{~Hz}, 1.0 \mathrm{~Hz}$ and $3.0 \mathrm{~Hz}$ groups was more up-regulated than the $5.0 \mathrm{~Hz}$ group compared to the control group. ADAMTS-4 expres- sion in the $1.0 \mathrm{~Hz}, 3.0 \mathrm{~Hz}$ and $5.0 \mathrm{~Hz}$ groups and MMP-3 expression in the $5.0 \mathrm{~Hz}$ group were all significantly up-regulated compared with the control group. In addition, TIMP-1 expression and TIMP-3 expression were increased significantly or not significantly in the $0.1 \mathrm{~Hz}, 0.5 \mathrm{~Hz}, 1 \mathrm{~Hz}$ and $3 \mathrm{~Hz}$ groups compared with the control group, whereas TIMP-3 expression in the $5.0 \mathrm{~Hz}$ group was significantly decreased.

(A) Compressive magnitude (Frequency: $1.0 \mathrm{~Hz}$; Duration: 2 hours/day)

Aggrecan
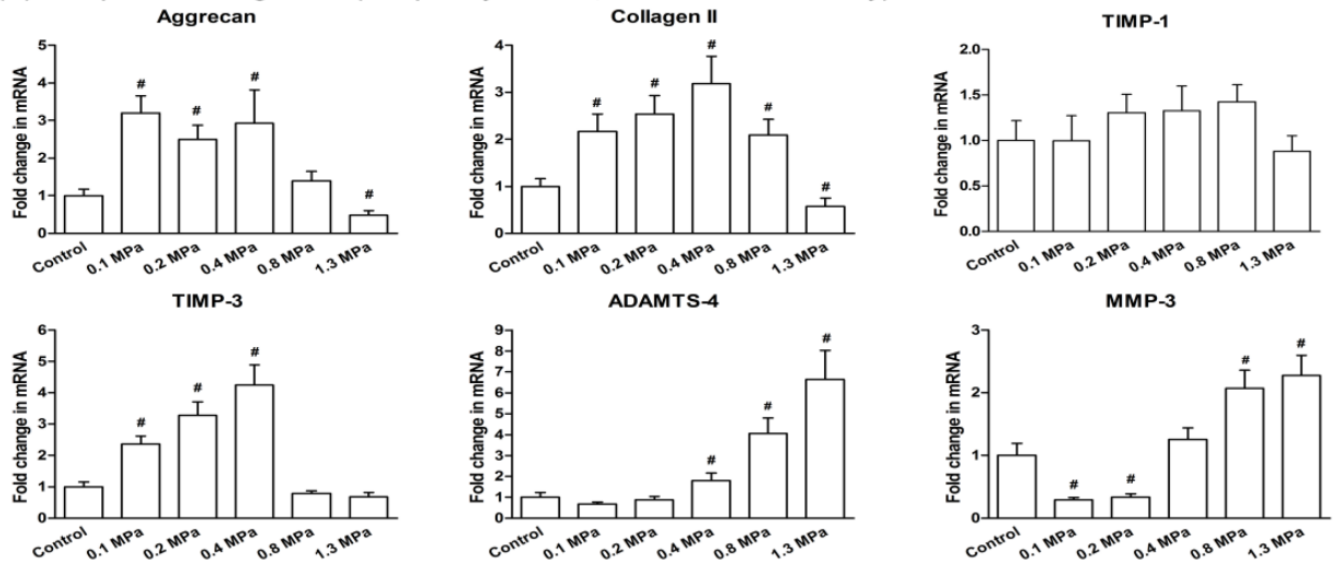

(B) Compressive frequency (Magnitude: 0.4 MPa; Duration: 2 hours/day)
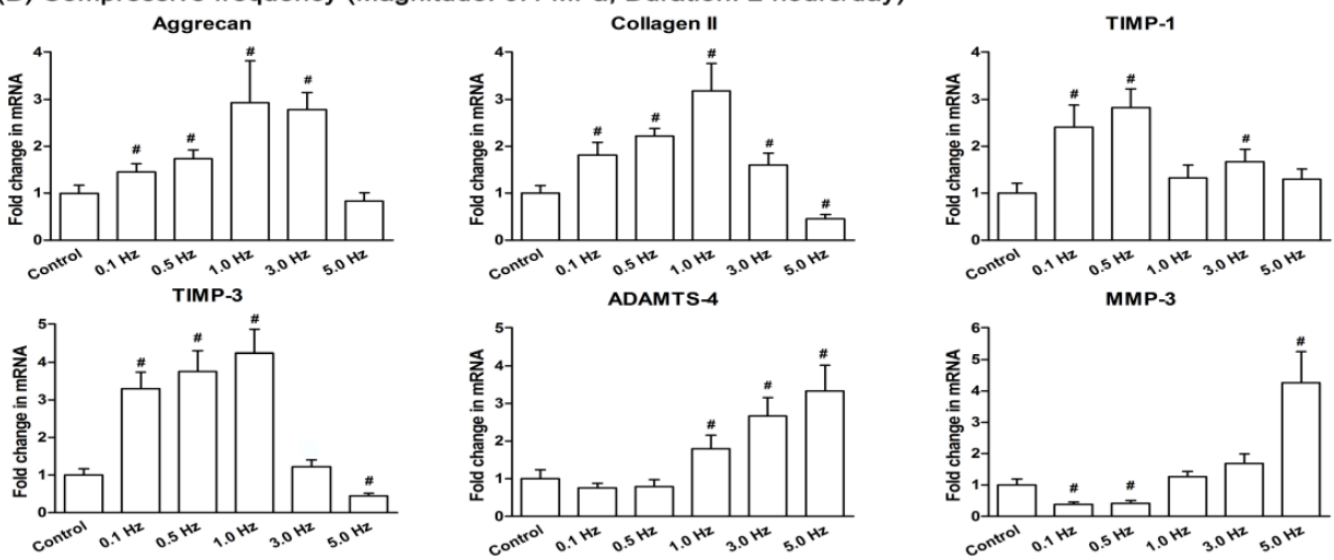

(C) Compressive duration (Magnitude: $0.4 \mathrm{MPa}$; Frequency: $1.0 \mathrm{~Hz}$ )
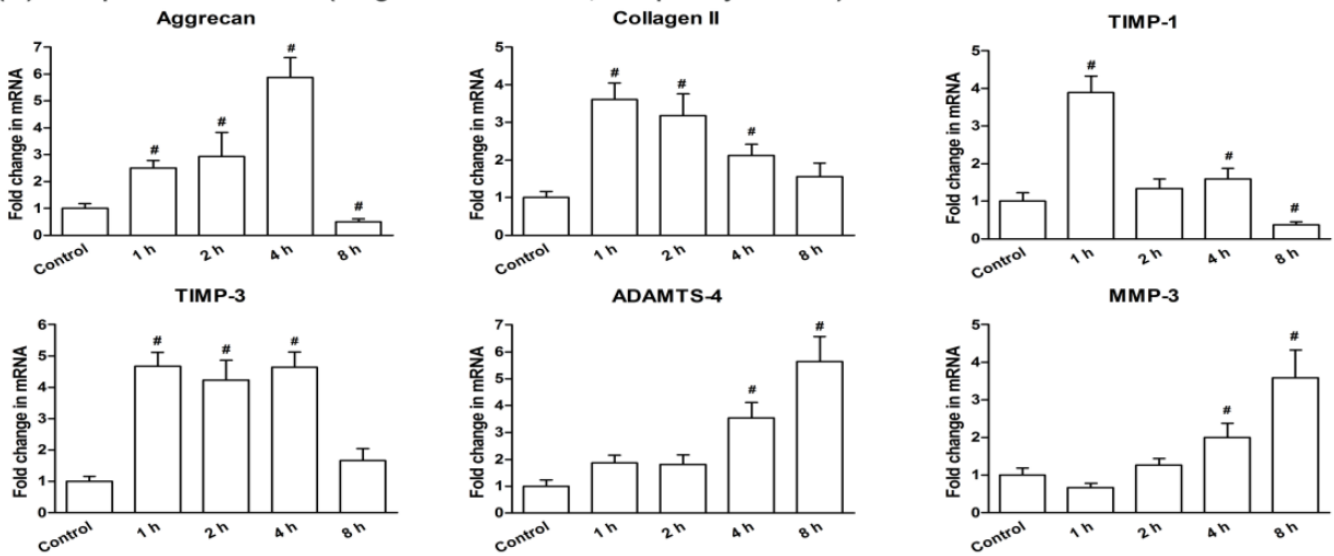

Figure 4. Anabolic (aggrecan, collagen II, TIMP-1 and TIMP-3) and catabolic (ADAMTS-4 and MMP-3) gene expression analysis. Gene expression of immature nucleus pulposus (NP) cells in different compressive magnitude groups (A), compressive frequency groups (B) and compressive duration groups (C). Data are expressed as the means $\pm S D, n=3$. \#: Indicates a significant difference $(p<0.05)$ compared with the control group $(p<0.05)$ between two groups. 
For the compressive duration groups (Figure $4 \mathrm{C})$, the expression of aggrecan, collagen II, TIMP-1 and TIMP-3 were all significantly or not significantly up-regulated, except the down-regulated expression of aggrecan and TIMP- 1 in the 8 hour group compared to the control group. Expression of both ADAMTS-4 and MMP-3 were up-regulated with increasing duration to a maximum in the 8 hour group.

\section{GAG content}

Figure 5 indicates that the GAG content was compressive magnitude-, frequency- and duration-dependent. In the $1.3 \mathrm{MPa}, 5.0 \mathrm{~Hz}$ and 8 hour groups, the GAG content was significantly decreased compared with the control group. For the compressive groups, a significantly or not significantly lower GAG content was observed in $1.3 \mathrm{MPa}, 5.0 \mathrm{~Hz}$ and 8 hour groups than the other compressive magnitude, frequency and duration groups.

\section{Collagen II protein expression}

Immunohistological staining showed that collagen II was differentially deposited in the various compressive groups. As shown in Figure 6, collagen II protein expression was decreased in the $1.3 \mathrm{MPa}, 5.0$ $\mathrm{Hz}$ and 8 hour groups and remained constant or increased in the other compressive groups compared with the control group.
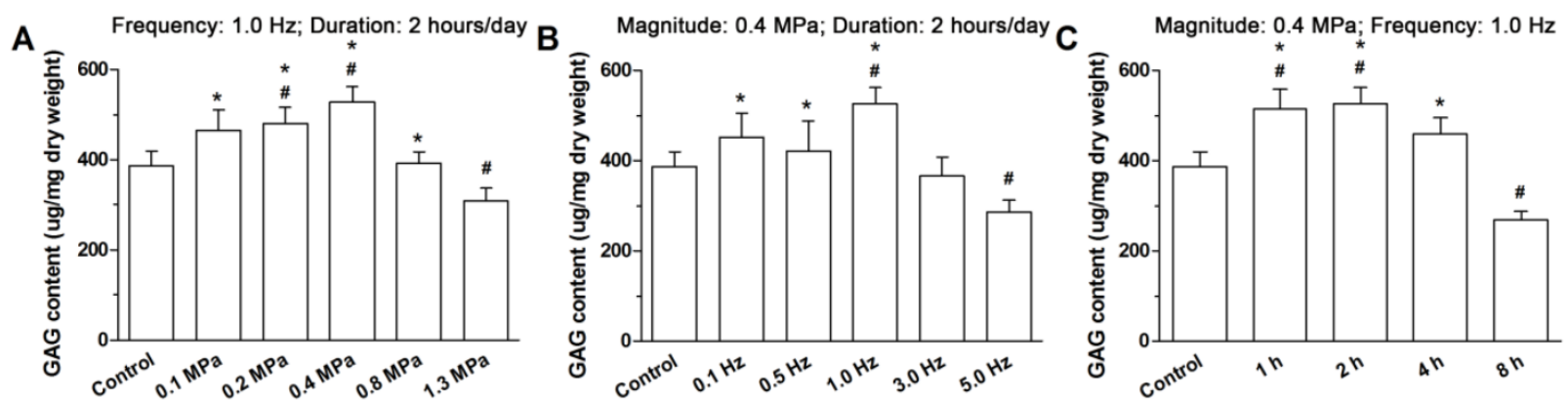

Figure 5. Glycosaminoglycan (GAG) content analysis. Immature nucleus pulposus (NP) samples are from discs cultured in different compressive magnitude groups $(A)$, compressive frequency groups $(B)$ and compressive duration groups $(C)$. Data are expressed as the means $\pm S D, n=3$. \#: Indicates a significant difference $(p<0.05$ ) compared with the control group $(\mathrm{p}<0.05)$ between two groups. *: Indicates a significant difference $(\mathrm{p}<0.05)$ compared with the compression groups $(1.3 \mathrm{MPa}, 5 \mathrm{~Hz}$ or 8 hour).

(A) Compressive magnitude (Frequency: $1.0 \mathrm{~Hz}$; Duration: 2 hours/day)

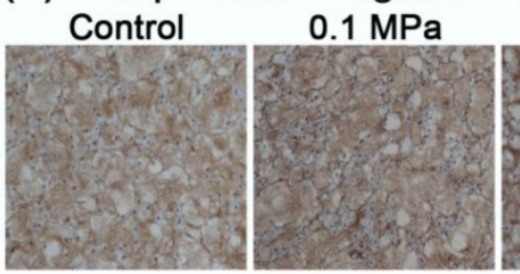

$0.2 \mathrm{MPa}$

$0.4 \mathrm{MPa}$

$0.8 \mathrm{MPa}$

1.3 MPa

(B) Compressive frequency (Magnitude: $0.4 \mathrm{MPa}$; Duration: 2 hours/day)

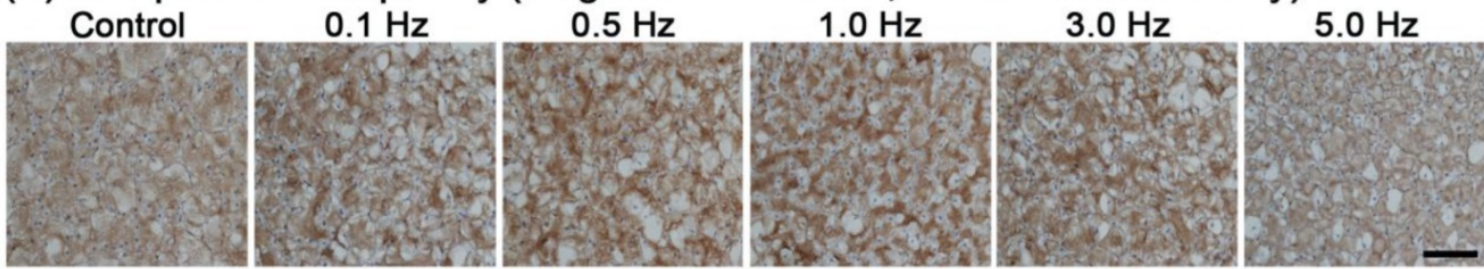

(C) Compressive duration (Magnitude: $0.4 \mathrm{MPa}$; Frequency: $1.0 \mathrm{~Hz}$ )

Control

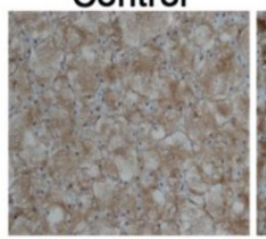

$1 \mathrm{~h}$

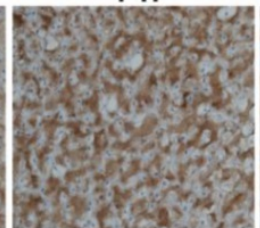

$4 \mathrm{~h}$

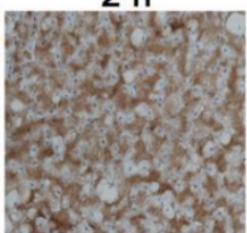

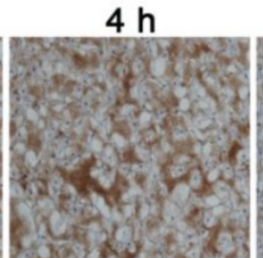

$8 \mathrm{~h}$

Figure 6. Representative photomicrographs of immunohistochemical staining of collagen II within the immature nucleus pulposus (NP). Results are analyzed from different compressive magnitude groups $(A)$, compressive frequency groups $(B)$ and compressive duration groups $(C)$. Magnification: $200 x ;$ scale $=100 \mu m ; n=3$. Data are expressed as the means $\pm S D, n=3$. 


\section{Discussion}

In the present study, we used an intelligent and mechanically active bioreactor to apply dynamic compression to the organ-cultured immature porcine discs. To our knowledge, few studies investigated the biological responses of immature porcine discs to mechanical compression in vitro. Our results showed that cell apoptosis and matrix composition within the immature NP depend on the compressive magnitude, frequency and duration, and a high compressive magnitude (1.3 $\mathrm{MPa})$, high compressive frequency $(5.0 \mathrm{~Hz})$ and longer compressive duration (8 hours) increased the apoptotic cells and decreased the matrix synthesis within the immature NP. This study may contribute to a better understanding of the mechanical compression-induced NP biological change in humans who are at a specific young age and ultimately the development of possible clinical strategies to prevent and/or restore IDD in the initiating stage.

Dynamic compression is commonly experienced during daily activities in vivo. The range of compressive magnitudes (0.1-1.3 $\mathrm{MPa}$ ), frequencies (0.1-5.0 $\mathrm{Hz}$ ) and durations (1-8 hours) were selected because they are within the human physiological situation [24, 25]. The immature porcine disc was used not only because of its feasibility in studying disc degeneration but also because of its high content of notochord cells, which resembles that of the immature human disc [26]. In addition, because the early pathological degenerative changes first occur in the NP region [27], we primarily focused on evaluating the biological changes of NP tissue in the present study.

Evidence from disc cell culture and animal in vivo studies indicates that the biological responses of disc cells to dynamic compression are magnitude dependent [28]. In our study, increased apoptotic NP cells, decreased biochemical content and a catabolic gene expression profile were found at 1.3 MP, suggesting that a relatively high compressive magnitude can increase cell apoptosis and decrease matrix biosynthesis within the immature NP. Consistent with our findings, Andrew et al. [7] also demonstrated that apoptotic disc cells increased with compressive magnitude in a murine tail model. However, a disc organ culture study by Korecki et al. [10] demonstrated that there were no differences in GAG content within the NP tissue from mature beef caudal discs between a low dynamic load group and high dynamic load group. This discrepancy may be due to the different cellular composition between the immature porcine $\mathrm{NP}$ and the mature beef NP. This difference also suggests that immature NP cells are more sensitive to mechanical stimuli than mature NP cells, which may be a reason for the disappearance of notochordal cells at an early age in humans [29]. Another significant finding of our study was the stable or even superior responses of immature NPs to dynamic compressive magnitudes less than $1.3 \mathrm{MPa}$, indicating that there is a magnitude threshold that can sustain cell viability and matrix homeostasis. This type of compressive magnitude threshold was also observed in previous studies [30, 31]. However, the difference in the magnitude threshold level exists between different studies, possibly due to the variation in tested parameters (mechanical or biological) and experimental design (organ culture, cell culture or in vivo study).

In addition to the compressive magnitude, the compressive frequency is another regulatory factor for disc cell biology [19]. In this study, we found that cell apoptosis and matrix synthesis within the immature NP were increased and inhibited in high compressive frequency group $(5.0 \mathrm{~Hz})$, respectively. Adarsh et al. [32] demonstrated that the resonant frequency at the lumbar spine is 4 to $6 \mathrm{~Hz}$. An epidemiological study by Wilder et al. [33] showed that dynamic loads with a frequency close to the spine resonant frequency have a destructive effect on disc biology. Here, the destructive effects of a $5.0 \mathrm{~Hz}$ frequency on cell viability and matrix composition within the immature NP directly support this finding. However, it is difficult to compare our study with other studies because mechanical compression-related studies on organ-cultured immature discs are relatively limited. Until now, only Wang et al. [6] studied effects of different compression frequencies on adolescent rabbit disc biology. This study demonstrated that dynamic frequency plays an important role in disc biosynthetic activity. However, only the effects of $0.1 \mathrm{~Hz}$ and $1.0 \mathrm{~Hz}$ at the same compression magnitude $(0.5 \mathrm{MPa}$ or $1.0 \mathrm{MPa})$ were studied in that study. Similarly, compressive frequency dependent effects on disc biology were also reported in some animal in vivo studies [7, 30, 34]. Despite the different experimental setups, we can establish that the maintenance of immature NP bioactivity may be achieved by the appropriate selection of the compression frequency.

In this study, we also investigated the responses of immature NPs to compressive duration. We found that non-apoptotic cells and matrix deposition (e.g. GAG content and collagen II protein expression) within the immature NP were decreased with compression duration. Moreover, catabolic genes (ADAMTS-4 and MMP-3) were significantly up-regulated in the long duration group (8 hours). These suggest that excessive daily exposure to dynamic compression leads to inferior cell viability and a disturbed metabolism within the immature NP. Consistent with this, an in vivo study by Lotz et al.[35] also demonstrated that the percentage of dead cells in 
the mouse tail disc was proportional to the time of spinal loading. These phenomena indicate that immature NP vitality can be improved with the appropriate daily compression exposure and destroyed with extremely extended daily exposure.

The notochordal cells contained in the immature NP tissue are regarded as the original cell population of the NP tissue, which can protect the disc from degeneration [15, 17, 36]. Previously, several cell markers were identified to distinguish notochordal NP cells from chondrocyte-like NP cells, such as galectin-3, cytokeratin-8, and E-cadherin [37-39]. Moreover, the notochordal NP cell phenotype can change into a chondrocyte-like NP cell phenotype under certain conditions, possibly associated with disc degeneration [18]. The porcine discs used in our study also contain many notochordal NP cells. We hypothesized that the cellular phenotype transformation may also occur and partially contribute to the degenerative changes in the high compressive magnitude, high compressive frequency and long compressive duration groups in this study. Consistent with this, a previous study also reported certain molecular changes at different stages of mechanically induced disc degeneration in an in vivo rabbit model [40]. This is an interesting and complex topic that needs further investigation.

This study also has some limitations. First, our results have limited stringency in reflecting the mechanobiology of the adult disc because the immature porcine disc contains a high content of notochordal cells. Second, previous cell culture studies and animal in vivo studies indicated that interactions between compression parameters could significantly affect disc cell biology $[8,30]$. Although we separately investigated the effects of compressive magnitude, compressive frequency or compressive duration on immature NPs, the interactions between these compression parameters were not studied. Third, possible mechanisms underlying the destructive effects of high compressive magnitude or compressive frequency and long compressive duration on immature NP biology were not studied here. In our future study, we will focus on the mechanism by which these destructive compressions lead to increased apoptotic cells and decreased matrix synthesis within the immature NP.

In conclusion, we studied the effects of dynamic compression on immature NPs in a disc bioreactor culture. Cell apoptosis and matrix composition within the immature NP were dependent on the compressive magnitude, frequency and duration. High compressive magnitude or frequency and long compressive duration led to increased apoptotic cells and decreased matrix composition within the immature NP.

\section{Acknowledgment}

We would like to thank Dr. Fuyun Ji for technical assistance. We also appreciated founding from the National Natural Science Foundation of China (NSFC 81272029 and NSFC 81027005), Science and Technology Achievement Transformation Fund of Third Military Medical University (2011XZH006).

\section{Conflicts}

The authors report no conflicts of interest.

\section{References}

1. Dagenais S, Caro J, Haldeman S. A systematic review of low back pain cost of illness studies in the United States and internationally. The spine journal : official journal of the North American Spine Society. 2008; 8: 8-20.

2. Luoma K, Riihimaki H, Luukkonen R, Raininko R, Viikari-Juntura E, Lamminen A. Low back pain in relation to lumbar disc degeneration. Spine. 2000; 25: 487-92.

3. Adams MA, Freeman BJ, Morrison HP, Nelson IW, Dolan P. Mechanical initiation of intervertebral disc degeneration. Spine. 2000; 25: 1625-36.

4. Hee HT, Zhang J, Wong HK. An in vitro study of dynamic cyclic compressive stress on human inner annulus fibrosus and nucleus pulposus cells. Spine J. 2010; 10: 795-801.

5. Fernando HN, Czamanski J, Yuan TY, Gu W, Salahadin A, Huang CY. Mechanical loading affects the energy metabolism of intervertebral disc cells. Journal of orthopaedic research : official publication of the Orthopaedic Research Society. 2011; 29: 1634-41.

6. Wang DL, Jiang SD, Dai LY. Biologic response of the intervertebral disc to static and dynamic compression in vitro. Spine. 2007; 32: 2521-8.

7. Walsh AJ, Lotz JC. Biological response of the intervertebral disc to dynamic loading. J Biomech. 2004; 37: 329-37.

8. Wuertz K, Godburn K, MacLean JJ, Barbir A, Donnelly JS, Roughley PJ, et al. In vivo remodeling of intervertebral discs in response to short- and long-term dynamic compression. Journal of orthopaedic research : official publication of the Orthopaedic Research Society. 2009; 27: 1235-42.

9. Jünger S, Gantenbein-Ritter B, Lezuo P, Alini M, Ferguson SJ, Ito K. Effect of limited nutrition on in situ intervertebral disc cells under simulated-physiological loading. Spine. 2009; 34: 1264-71.

10. Korecki CL, MacLean JJ, Iatridis JC. Dynamic compression effects on intervertebral disc mechanics and biology. Spine. 2008; 33: 1403-9.

11. Chan SC, Ferguson SJ, Wuertz K, Gantenbein-Ritter B. Biological response of the intervertebral disc to repetitive short-term cyclic torsion. Spine. 2011; 36: 2021-30.

12. Li ST, Liu Y, Zhou Q, Lue RF, Song L, Dong SW, et al. A novel axial-stress bioreactor system combined with a substance exchanger for tissue engineering of 3D constructs. Tissue Eng Part C Methods. 2014; 20: 205-14.

13. Haglund L, Moir J, Beckman L, Mulligan KR, Jim B, Ouellet JA, et al. Development of a bioreactor for axially loaded intervertebral disc organ culture. Tissue Eng Part C Methods. 2011; 17: 1011-9.

14. Urban JP. The role of the physicochemical environment in determining disc cell behaviour. Biochemical Society transactions. 2002; 30: 858-64.

15. Nerlich AG, Schleicher ED, Boos N. 1997 Volvo Award winner in basic science studies. Immunohistologic markers for age-related changes of human lumbar intervertebral discs. Spine (Phila Pa 1976). 1997; 22: 2781-95.

16. T R O. The role of disc cell heterogeneity in determining disc biochemistry: a speculation. Biochem Soc Trans. 2002; 30: 839-44.

17. Guehring T, Nerlich A, Kroeber M, Richter W, Omlor GW. Sensitivity of notochordal disc cells to mechanical loading: an experimental animal study. European spine journal : official publication of the European Spine Society, the European Spinal Deformity Society, and the European Section of the Cervical Spine Research Society. 2010; 19: 113-21.

18. Omlor GW, Nerlich AG, Hans-Joachim W, Michael P, Helga L, Markus SK, et al. A new porcine in vivo animal model of disc degeneration: response of anulus fibrosus cells, chondrocyte-like nucleus pulposus cells, and notochordal nucleus pulposus cells to partial nucleotomy. Spine. 2009; 34 : 2730-9.

19. Palmer EI, Lotz JC. The compressive creep properties of normal and degenerated murine intervertebral discs. Journal of orthopaedic research : official publication of the Orthopaedic Research Society. 2004; 22: 164-9.

20. Lee CR, Iatridis JC, Poveda L, Alini M. In vitro organ culture of the bovine intervertebral disc: effects of vertebral endplate and potential for mechanobiology studies. Spine. 2006; 31: 515-22.

21. Beckstein JC, Sen S, Schaer TP, Vresilovic EJ, Elliott DM. Comparison of animal discs used in disc research to human lumbar disc: axial compression mechanics and glycosaminoglycan content. Spine. 2008; 33: E166-73. 
22. Farndale RW, Sayers CA, Barrett AJ. A direct spectrophotometric microassay for sulfated glycosaminoglycans in cartilage cultures. Connect Tissue Res. 1982; 9: 247-8.

23. Le Maitre CL, Freemont AJ, Hoyland JA. The role of interleukin-1 in the pathogenesis of human intervertebral disc degeneration. Arthritis research \& therapy. 2005; 7: R732-45.

24. Urban JP, McMullin JF. Swelling pressure of the lumbar intervertebral discs: influence of age, spinal level, composition, and degeneration. Spine. 1988; 13: 179-87.

25. Pope MH, Magnusson M, Wilder DG. Kappa Delta Award. Low back pain and whole body vibration. Clin Orthop Relat Res. 1998: 241-8.

26. Chen J, Yan W, Setton LA. Molecular phenotypes of notochordal cells purified from immature nucleus pulposus. European spine journal : official publication of the European Spine Society, the European Spinal Deformity Society, and the European Section of the Cervical Spine Research Society. 2006; 15 Suppl 3: S303-11.

27. Clouet J, Vinatier C, Merceron C, Pot-Vaucel M, Hamel O, Weiss P, et al. The intervertebral disc: from pathophysiology to tissue engineering. Joint, bone, spine : revue du rhumatisme. 2009; 76: 614-8.

28. Chan SC, Ferguson SJ, Gantenbein-Ritter B. The effects of dynamic loading on the intervertebral disc. European spine journal : official publication of the European Spine Society, the European Spinal Deformity Society, and the European Section of the Cervical Spine Research Society. 2011; 20: 1796-812.

29. Korecki CL, Kuo CK, Tuan RS, Iatridis JC. Intervertebral disc cell response to dynamic compression is age and frequency dependent. Journal of Orthopaedic Research. 2009; 27: 800-6.

30. Maclean JJ, Lee CR, Alini M, Iatridis JC. Anabolic and catabolic mRNA levels of the intervertebral disc vary with the magnitude and frequency of in vivo dynamic compression. Journal of orthopaedic research : official publication of the Orthopaedic Research Society. 2004; 22: 1193-200.

31. Ching CT, Chow DH, Yao FY, Holmes AD. The effect of cyclic compression on the mechanical properties of the inter-vertebral disc: an in vivo study in a rat tail model. Clinical biomechanics (Bristol, Avon). 2003; 18: 182-9.

32. Kumar A, Varghese M, Mohan D, Mahajan P, Gulati P, Kale S. Effect of whole-body vibration on the low back. A study of tractor-driving farmers in north India. Spine. 1999; 24: 2506-15.

33. Wilder DG, Pope MH. Epidemiological and aetiological aspects of low back pain in vibration environments - an update. Clinical biomechanics (Bristol, Avon). 1996; 11: 61-73.

34. Ching CT, Chow DH, Yao FY, Holmes AD. Changes in nuclear composition following cyclic compression of the intervertebral disc in an in vivo rat-tail model. Medical engineering \& physics. 2004; 26: 587-94.

35. Lotz JC, Chin JR. Intervertebral disc cell death is dependent on the magnitude and duration of spinal loading. Spine. 2000; 25: 1477-83.

36. Hunter CJ, Matyas JR, Duncan NA. Cytomorphology of notochordal and chondrocytic cells from the nucleus pulposus: a species comparison. Journal of anatomy. 2004; 205: 357-62.

37. Oguz E, Tsai TT, Di Martino A, Guttapalli A, Albert TJ, Shapiro IM, et al. Galectin-3 expression in the intervertebral disc: a useful marker of the notochord phenotype? Spine. 2007; 32: 9-16.

38. Lv F, Leung VY, Huang S, Huang Y, Sun Y, Cheung KM. In search of nucleus pulposus-specific molecular markers. Rheumatology. 2014; 53: 600-10.

39. Weiler C, Nerlich AG, Schaaf R, Bachmeier BE, Wuertz K, Boos N. Immunohistochemical identification of notochordal markers in cells in the aging human lumbar intervertebral disc. European spine journal : official publication of the European Spine Society, the European Spinal Deformity Society, and the European Section of the Cervical Spine Research Society. 2010; 19: $1761-70$.

40. Omlor GW, Lorenz H, Engelleiter K, Richter W, Carstens C, Kroeber MW, et al. Changes in gene expression and protein distribution at different stages of mechanically induced disc degeneration--an in vivo study on the New Zealand white rabbit. Journal of orthopaedic research : official publication of the Orthopaedic Research Society. 2006; 24: 385-92. 